\title{
Diagnostic yield of specific inhalation challenge in hypersensitivity pneumonitis
}

\author{
Xavier Muñoz ${ }^{1,2,3}$, Mónica Sánchez-Ortiz',2, Ferran Torres ${ }^{4}$, Ana Villar ${ }^{1,2}$, \\ Ferran Morell ${ }^{1,2}$ and María-Jesús Cruz ${ }^{1,2}$
}

\author{
Affiliations: \\ 'Pulmonology Service, Medicine Dept, Hospital Universitari Vall d’Hebron, Universitat Autònoma de Barcelona, \\ Barcelona, Spain. \\ ${ }^{2}$ CIBER Enfermedades Respiratorias (Ciberes), Spain. \\ ${ }^{3}$ Dept of Cell Biology, Physiology and Immunology, Universitat Autònoma de Barcelona, Barcelona, Spain. \\ ${ }^{4}$ Biostatistics and Data Management Platform, IDIBAPS, Hospital Clinic, Biostatistics Unit, School of Medicine, \\ Universitat Autònoma de Barcelona, Barcelona, Spain.
}

\section{Correspondence:}

Xavier Muñoz, Servei de Pneumologia, Hospital Universitari Vall d'Hebron, Passeig Vall d'Hebron, 119, 08035 Barcelona, Spain.

E-mail: xmunozQvivebron.net

ABSTRACT Reliable methods are needed to diagnose hypersensitivity pneumonitis. The aim of the study was to establish the diagnostic yield of specific inhalation challenge (SIC) in patients with hypersensitivity pneumonitis.

All patients with suspected hypersensitivity pneumonitis in whom SIC was performed $(n=113)$ were included. SIC was considered positive when patients showed a decrease of $>15 \%$ in forced vital capacity (FVC) or $>20 \%$ in diffusing capacity of the lung for carbon dioxide, or a decrease of $10 \%$ to $15 \%$ in FVC accompanied by a temperature increase of $0.5^{\circ} \mathrm{C}$ within $24 \mathrm{~h}$ of inhalation of the antigen.

SIC was positive to the agents tested in 68 patients: 64 received a diagnosis of hypersensitivity pneumonitis and SIC results were considered false-positive in the remaining four patients. In the SICnegative group $(n=45), 24$ patients received a diagnosis of hypersensitivity pneumonitis and SIC results were considered false-negative, and 21 patients were diagnosed with other respiratory diseases. The sensitivity and specificity of the test were $72.7 \%$ and $84 \%$, respectively. Having hypersensitivity pneumonitis caused by an antigen other than birds or fungi predicted a false-negative result $(p=0.001)$.

In hypersensitivity pneumonitis, positive SIC testing virtually confirms the diagnosis, whereas negative testing does not rule it out, especially when the antigenic sources are not birds or fungi.

@ERSpublications

Specific inhalation challenge can be very useful for establishing the diagnosis of hypersensitivity pneumonitis http://ow.ly/zxszi (FUCAP, Catalonian Pulmonology Foundation). M.J. Cruz is a researcher supported by the Miguel Servet programme from Instituto de Salud Carlos III (CP12/03101). The funders had no role in the study design, data collection or analysis, decision to publish, or preparation of the manuscript.

Conflict of interest: None declared. 


\section{Introduction}

Hypersensitivity pneumonitis is a disease with heterogeneous causes, resulting from an inflammatory pulmonary reaction of immunological origin in response to a wide variety of antigens that can provoke varying degrees of inflammation and destructuring of the lung parenchyma [1]. Currently, most authors agree that the diagnosis of hypersensitivity pneumonitis should be based on the criteria proposed by SCHUyler and CORMIER [2]. The development and clinical presentation of hypersensitivity pneumonitis are influenced by several factors [3-6]. Genetic susceptibility may explain why one individual develops the disease, another individual with exactly the same exposure is only sensitised but remains healthy, and yet another will not even become sensitised $[3,4]$. In this context, in which a process of sensitisation seems essential, the specific inhalation challenge (SIC) could be a tool for diagnosing this condition [5], especially bearing in mind the immunological and histopathological response elicited in affected patients [6].

Nevertheless, SIC currently lacks standardisation. Only four studies to date, all in the framework of bird fancier's lung, have attempted to establish the performance of the test [7-10]. The sensitivity and specificity values obtained in these studies were between $80 \%$ and $100 \%$, but the small number of patients tested and, in particular, the fact that the manner of performing and interpreting the test substantially differed between studies may be the reasons why this diagnostic tool is not more widely used.

The aim of this study was to evaluate the performance of SIC for diagnosing hypersensitivity pneumonitis due to a variety of causal agents in a large number of patients with this condition.

\section{Materials and methods \\ Study population}

This was a retrospective, cross-sectional study including all patients older than 18 years with suspected hypersensitivity pneumonitis who underwent SIC in our centre (Hospital Universitari Vall d'Hebron, Barcelona, Spain) between June 1995 and December 2010. Some of these patients had been initially seen at other hospitals and were later referred to our centre to complete the diagnostic study.

\section{Diagnostic protocol}

In all patients, we analysed data from the clinical history and physical examination, as well as the results from the following additional tests: laboratory analyses with complete blood count, erythrocyte sedimentation rate, gammaglobulin, total G and E immunoglobulins, calcium level, 24-h urinary calcium, plasma angiotensin-converting enzyme and lactate dehydrogenase levels, as well as specific serum immunoglobulin $\mathrm{G}$ antibody determinations, chest radiograph, chest computed tomography, pulmonary function testing including spirometry, static lung volumes, and carbon monoxide diffusing capacity (DLCO), immediate and delayed hypersensitivity skin testing, bronchofibroscopy with bronchoalveolar lavage and/or transbronchial biopsy, and SIC. In the few cases in which a definite diagnosis could not be reached once these tests had been carried out, surgical lung biopsy was performed after individualised assessment of the indication [10].

\section{Diagnostic confirmation}

The definite diagnosis of hypersensitivity pneumonitis was established based on independent review of the patients' medical records by two pulmonologists with extensive experience in diffuse interstitial lung disease who were unaware of the SIC results. In addition to evaluation of the data at the time when SIC was performed, the two experts also assessed the patients' clinical follow-up data up to the time of writing. The diagnosis of hypersensitivity pneumonitis was established by the two specialists according to the same diagnostic protocol [10] based on the proposed criteria of SCHUYLER and CORMIER [2].

\section{Antigen extract preparation for specific inhalation challenge}

Commercialised extracts (Bial-Aristegui, Bilbao, Spain) from Penicillium frequentans, Aspergillus fumigatus and Mucor mucedo were used to study fungi [11]. The avian sera were prepared in our laboratory. Blood for avian serum extracts was collected from several birds and centrifuged; the serum protein concentration was measured by bicinchoninic acid assay (Pierce Chemicals, Rockford, IL, USA). The extracts for SIC to isocyanates and esparto were prepared as previously described [11, 12]. The SIC to metalworking fluid, dental prostheses and interferon were performed with the material provided by the patient to simulate the working conditions in a challenge room.

\section{Specific inhalation challenge}

SIC was performed in the hospital setting after obtaining the patient's written consent. In patients receiving anti-inflammatory medication (corticosteroids), the medication was discontinued at least 8 days before SIC was carried out. Patients were required to have a baseline forced vital capacity (FVC) $>50 \%$ and 
DLCO $\geqslant 40 \%$ of the reference value [13] to be considered for SIC testing. The test was performed on an outpatient basis.

In 81 patients, SIC consisted of nebulisation of an extract of the suspected causal agent using a De Vilbiss 646 nebuliser (De Vilbiss CO, Somerset, PA, USA) from 1995 to 2001 or a Mefar MB3 dosimeter (Mefar, Ele $\mathrm{H}_{2} \mathrm{O}$; Medicali, Brescia, Italy) from 2001 to 2010, which release the solution during the first second of each inspiration. Patients were requested to inhale $2 \mathrm{~mL}$ of the suspected antigen at dilution of 1:100 $\left(0.01 \mathrm{mg} \cdot \mathrm{mL}^{-1}\right)[10,13]$. FVC, forced expiratory volume in $1 \mathrm{~s}$, DLCO, and the patient's temperature were recorded at baseline, $20 \mathrm{~min}$ after inhalation challenge, every hour thereafter for the next $8 \mathrm{~h}$ and at $24 \mathrm{~h}$. Blood cell count, chest radiograph and $\mathrm{O}_{2}$ saturation measurement were performed before and $8 \mathrm{~h}$ after inhalation. In all cases, SIC with a placebo solution (saline) was carried out 1 day before testing with the suspected antigen. None of the patients responded positively to the placebo solution.

The test was considered positive when any of the following responses was elicited [10, 13]. 1) FVC decrease of $>15 \%$ or DLCO decrease of $>20 \%$ as compared to baseline values. 2) $10 \%$ to $15 \%$ FVC decrease plus at least one of the following five criteria with respect to clinical status and baseline analytical values: white blood cell increase of $20 \% ; \mathrm{O}_{2}$ saturation decrease of $3 \%$; significant radiological changes; rise in body temperature of more than $0.5^{\circ} \mathrm{C}$; or evident clinical symptoms (e.g. cough or dyspnoea). 3) FVC decrease $<10 \%$ but with evidence of three or more of the previously mentioned clinical or analytical criteria. When the test proved negative, inhalation of a new antigen dilution of $1: 10\left(0.1 \mathrm{mg} \cdot \mathrm{mL}^{-1}\right)$ was performed the next day following the same procedure.

In 32 patients, the antigens used were not soluble (isocyanates, wood dust, humidifiers, duvets, etc.), and the SIC was conducted by directly exposing the patient to the suspected causal agent in a challenge chamber $[12,14,15]$. When the previous result was negative, the exposure time was increased on successive days, up to a maximum exposure of $2 \mathrm{~h}$ per day. The clinical and functional response and the criteria to establish positivity were those mentioned above.

\section{Pulmonary function testing}

Pulmonary function testing was carried out on a MasterLab system (MasterLab, Jaeger, Germany) according to the guidelines of the European Respiratory Society [16, 17]. The forced spirometry reference values were those proposed for the Mediterranean population [18]. For the diffusion study, the singlebreath diffusing capacity of the lung for carbon monoxide was used. The theoretical values were those proposed by the European Respiratory Society [17]. Starting in 2006, the European Respiratory Society/ American Thoracic Society joint guidelines were used $[19,20]$.

\section{Statistical analysis}

The Mann-Whitney test and chi-squared test were applied to compare continuous and nominal variables, respectively, with a two-sided significance level of 0.05 . The consistency of SIC was estimated by evaluating the sensitivity and specificity [21] of the method, the positive and negative predictive values [22], and the likelihood ratio of a positive and negative value [23] with $95 \%$ confidence intervals using the Wilson method [24]. Receiver operating characteristic (ROC) curves were constructed to determine the cut-off values that best differentiated between having the disease or not [25]. All analyses were done with SPSS, version 17 (Chicago, IL, USA) and SAS version 9.2 (SAS Institute Inc., Cary, NC, USA).

\section{Results}

Of the original 136 patients with suspected hypersensitivity pneumonitis referred for SIC, 23 were excluded. The test was not performed in four patients due to a DLCO $<40 \%$, and in the other 19 no clinical data were available. Among the 113 subjects undergoing the test, SIC was positive to the agents tested in 68 patients, 64 of whom were diagnosed with hypersensitivity pneumonitis (group 1). The SIC was considered to yield false-positive results in the four remaining patients (group 2), who were diagnosed with smoking-related bronchiolitis, cryptogenic organising pneumonia due ortho-phenylenediamine exposure [26], sarcoidosis and idiopathic pulmonary haemosiderosis. In the SIC-negative group $(n=45), 24$ patients received a diagnosis of hypersensitivity pneumonitis and SIC results were considered false-negative (group 3), and 21 patients were diagnosed with other respiratory diseases: chronic obstructive pulmonary disease $(n=5)$, idiopathic pulmonary fibrosis $(n=4)$, bronchiectasis $(n=4)$, bronchiolitis $(n=3)$, silicosis $(n=1)$, microscopic polyangiitis $(n=1)$, sarcoidosis $(n=1)$, nonspecific interstitial pneumonia $(n=1)$, and asthma $(n=1)$ (group 4) (fig. 1). None of the patients in group 4 presented symptoms or impaired lung function during SIC.

Demographic data and clinical characteristics of the total series and the group 1 and group 3 patients are summarised in table 1 . Patient age and percentage of nonsmokers were significantly higher in group 3 than 


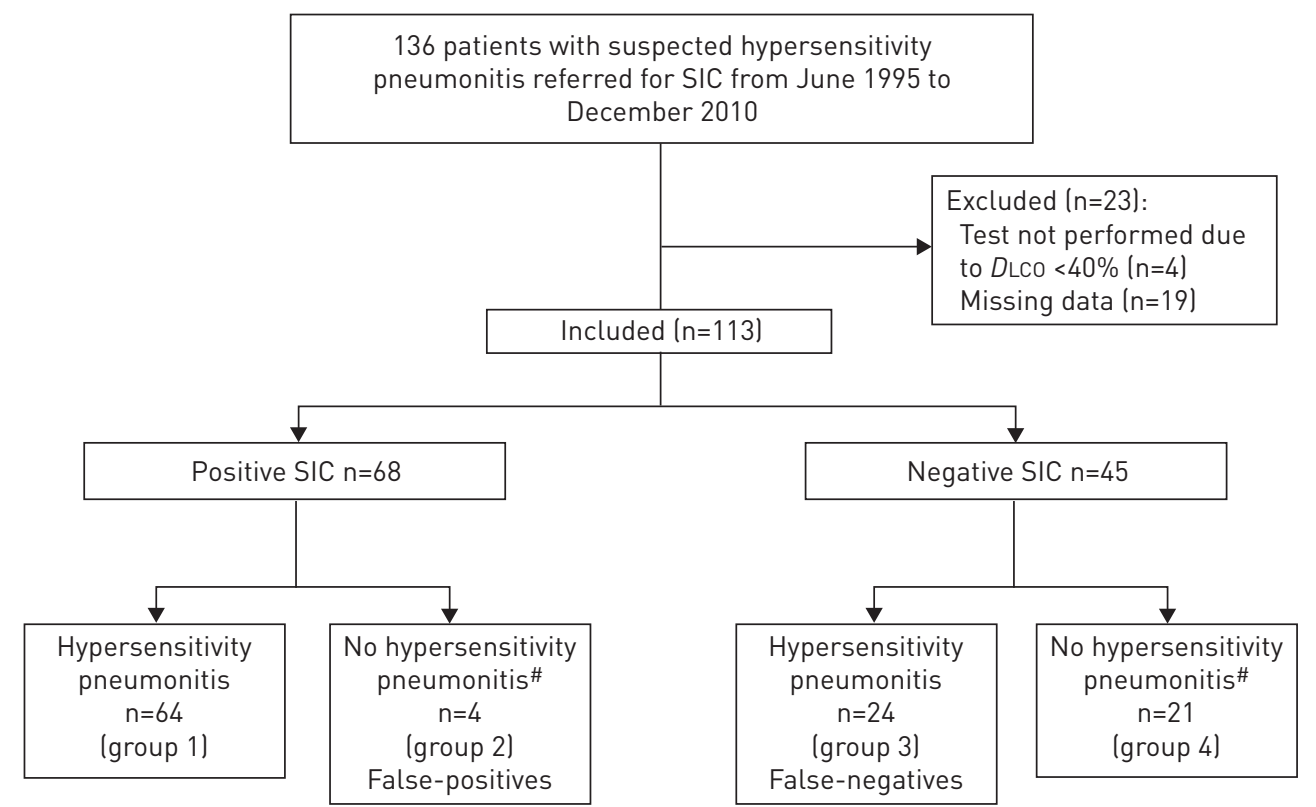

FIGURE 1 Flow chart of patient enrolment. SIC: specific inhalation challenge; DLCO: diffusing capacity of the lung for carbon monoxide. ${ }^{\#}$ : see diagnostics in text.

in group 1 ( $\mathrm{p}=0.004$ and $\mathrm{p}=0.003$, respectively). In group $3,58.3 \%$ of the hypersensitivity pneumonitis cases were not caused by avian or fungal proteins, whereas in group 1 only $11 \%$ of cases were not caused by these agents $(\mathrm{p}<0.001)$. The non-avian non-fungal agents causing these cases were isocyanates $(n=3)$; esparto $(n=2)$; wood $(n=2)$ metalworking fluid $(n=1)$, dental prosthesis $(n=1)$ and interferon $(n=1)$. In 11 patients, the causal antigen was not found. In these 11 patients, SIC was performed to several suspected antigens, but no positive results were recorded.

The sensitivity, specificity, and positive and negative predictive values of SIC according to the established criteria for positive status (FVC, DLCO and temperature) are shown in table 2, with 95\% confidence intervals. The sensitivity and negative predictive value of the test were higher when only patients with hypersensitivity pneumonitis caused by avian or fungal proteins were assessed (table 2). The ROC curves in figure 2 show the most relevant differences in FVC, DLCO and temperature alone before and after SIC for predicting positive SIC status. Using the ROC curve, we found that a more than $10 \%$ FVC decrease achieved the most satisfactory sensitivity $(45.5 \%, 95 \%$ CI 35.5-55.8) and specificity (88\%, 95\% CI 70-95.8) for the diagnosis of hypersensitivity pneumonitis. For DLCO, a post-SIC decrease greater than $15 \%$ resulted in a sensitivity of $45.5 \%$ ( $95 \%$ CI 35.5-55.8) and specificity of 76\% (95\% CI 56.6-88.5). Regarding temperature, an increase higher than $0.5^{\circ} \mathrm{C}$ after SIC achieved a sensitivity of $37.5 \%$ (95\% CI 28.1-47.9) and specificity of $72 \%$ (95\% CI 52.4-85.7).

Nine patients $(8 \%)$ experienced severe reactions related to SIC testing. These included influenza-like symptoms in all cases, with fever up to $40^{\circ} \mathrm{C}$ in one patient, hypoxaemia with an arterial oxygen tension $\left(\mathrm{PaO}_{2}\right)$ decrease of $10 \mathrm{mmHg}$ in another patient, and the development of new radiological changes in another. All these severe reactions were transient, and only the three patients mentioned required administration of oral corticosteroids. None required hospital admission. As compared to patients who did not present severe reactions, these nine patients were younger (mean \pm SD age $39.56 \pm 13.07$ versus $53.29 \pm 14.48$ years; $\mathrm{p}=0.009)$ and pre-SIC DLCO was higher $(94.28 \pm 17.44 \%$ versus $69.67 \pm 21.17 \%$ of theoretical value; $\mathrm{p}=0.005)$. There were no significant differences in the remaining variables analysed.

\section{Discussion}

The results of this study show that SIC could be useful as a tool for diagnosing hypersensitivity pneumonitis, with a positive predictive value of $94 \%$ and negative predictive value of $46.6 \%$ when considering the overall tests performed, and negative predictive value of $74.4 \%$ when including only tests performed with avian or fungal antigens. Of note, the likelihood ratios for positive and negative values, which are independent of the high prevalence observed in our sample, were $>5$ and $<2$, respectively. This is clearly an indication of the high utility of the combined diagnostic test to differentiate between patients testing positive and negative [23]. To our knowledge, this is the first study assessing the diagnostic yield of 
TABLE 1 Demographic data and clinical characteristics of the study subjects

\begin{tabular}{|c|c|c|c|}
\hline & Total & Group 1 & Group 3 \\
\hline Subjects & 113 & 64 & 24 \\
\hline Age years & $52 \pm 14.8$ & $50 \pm 14.6^{\# \#}$ & $59.5 \pm 13.8^{\# \#}$ \\
\hline Male & $49(43.4)$ & $28(43.8)$ & $10(41.7)$ \\
\hline Exposure time (months) before SIC & $170 \pm 168$ & $171 \pm 167$ & $159.6 \pm 179$ \\
\hline Duration of symptoms (months) before SIC & $37.2 \pm 45.5$ & $42.5 \pm 52.5$ & $22.82 \pm 19.2$ \\
\hline $\begin{array}{l}\text { Time (months) since last exposure } \\
\text { Smoking habit }\end{array}$ & $10.6 \pm 31.8$ & $12.3 \pm 38.9$ & $4.2 \pm 7.8$ \\
\hline Smoker & $19(18.1)$ & $13(22)$ & $2(9.1)$ \\
\hline Nonsmoker & $56(53.3)$ & $24(40.7)$ & 18 (81.8) \\
\hline Ex-smoker & $30(28.6)$ & 22 (37.3) & $2(9.1)$ \\
\hline Lymphocytes in BAL \% & $22.4 \pm 21.3$ & $25 \pm 23.7$ & $24 \pm 18.9$ \\
\hline FVC before SIC \% & $79.3 \pm 16.6$ & $78.75 \pm 18.2$ & $75.7 \pm 13.8$ \\
\hline FEV1 before SIC \% & $86.4 \pm 18.2$ & $83.60 \pm 18.0$ & $88 \pm 18.8$ \\
\hline FEV1/FVC before SIC \% & $81.3 \pm 13.8$ & $81.6 \pm 8.8$ & $82.2 \pm 19.0$ \\
\hline TLC before SIC \% & $86.9 \pm 16.6$ & $86.4 \pm 19.1$ & $85.7 \pm 12.0$ \\
\hline DLco before SIC \% & $71.3 \pm 21.7$ & $70 \pm 23.4$ & $71.2 \pm 18.9$ \\
\hline \multicolumn{4}{|l|}{ Chest CT } \\
\hline Normal & $10(9.6)$ & $6(10)$ & $2(9.1)$ \\
\hline Nodular & $15(14.4)$ & $11(18.3)$ & $1(4.5)$ \\
\hline Reticular & $5(4.8)$ & $3(5)$ & $2(9.14 .7)$ \\
\hline UIP & 19 (18.3) & $9(15)$ & $7(31.8)$ \\
\hline Ground glass & 39 (37.5) & $24(40)$ & $10(45.5)$ \\
\hline Emphysema & $6(5.8)$ & $2(3.3)$ & 0 \\
\hline Bronchiectasis & $10(9.6)$ & $5(8.3)$ & 0 \\
\hline \multicolumn{4}{|l|}{ Antigen SIC } \\
\hline Avian proteins & 65 (57.5) & $34(53.1)$ & $17(70.8)$ \\
\hline Fungi & $30(26.5)$ & $23(35.9)$ & $4(16.7)$ \\
\hline Others & $18(15.9)$ & $7(10.9)$ & $3(12.5)$ \\
\hline \multicolumn{4}{|l|}{ Final clinical diagnosis*** } \\
\hline HP due to avian proteins & $43(38.1)$ & $34(53.1)$ & $9(37.5)$ \\
\hline HP due to fungi & $24(21.2)$ & $23(35.9)$ & $1(4.2)$ \\
\hline HP due to other antigens & $21(18.6)$ & $7(10.9)$ & 14 (58.3) \\
\hline No HP & $25(22.1)$ & & \\
\hline
\end{tabular}

Data are presented as $\mathrm{n}, \mathrm{n}(\%)$, or mean \pm SD. SIC: specific inhalation challenge; BAL: bronchoalveolar lavage; FVC: forced vital capacity; FEV1: forced expiratory volume in $1 \mathrm{~s}$; TLC: total lung capacity; DLCo: diffusing capacity of the lung for carbon monoxide; CT: computed tomography; UIP: usual interstitial pneumonia; HP: hypersensitivity pneumonitis. ${ }^{\# \#}: p=0.004 ;{ }^{\text {ศศ }}: p=0.003$, group 1 versus group $3{ }^{* * *}: p<0.001$.

SIC in a clinical practice setting in a large number of cases and with no restrictions on the agents causing hypersensitivity pneumonitis.

Although SIC has been used for the diagnosis of hypersensitivity pneumonitis since the 1960s, it was not until 1980 that HENDRICK et al. [7] attempted to determine the performance of this test in a study including 29 cases of suspected hypersensitivity pneumonitis due to avian antigens. The authors reported a sensitivity of $48 \%$ to $85 \%$ and a specificity of $95 \%$. Also in the context of hypersensitivity pneumonitis due to avian antigens, RAMírEZ-VENEGAS et al. [8] studied 17 patients with chronic hypersensitivity pneumonitis and 17 patients with other interstitial lung diseases and reported a sensitivity of $82 \%$ to $86 \%$ and specificity of $76 \%$ to $100 \%$, whereas OHTANi et al. [9] found no false results in a study of 11 patients with bird fancier's lung and six control subjects. The largest study to date, also conducted in patients with bird fancier's lung, showed a sensitivity and specificity of $92 \%$ and $100 \%$, respectively, in 59 patients with hypersensitivity pneumonitis, 30 healthy pigeon keepers, and 20 patients with other diffuse interstitial lung diseases [10].

One noteworthy finding in the present study was the elevated number of false-negative test results. In 24 patients with hypersensitivity pneumonitis, SIC results were negative. The first reason for this may be related to the fact that our study was based on regular clinical practice, whereas in the ones mentioned above [7-9], the study protocol may have had an effect on the results. In this sense, it may not have been completely appropriate to include as false-negative cases the 11 patients in whom the causal agent was not found. It may have been that the antigen used for SIC was simply not the one causing the disease. Second, 
TABLE 2 Diagnostic performance of specific inhalation challenge (SIC)

All SIC

113

Subjects $\mathbf{n}$

Prevalence \%

Sensitivity \%

Specificity $\%$

PPV \%

NPV \%

$\mathrm{LR}+\%$

LR- \%
SIC to avian proteins and fungi

$\begin{array}{cc}113 & 95 \\ 78 & 73 \\ 72.7(62.6-80.9) & 85.1(74.7-91.7) \\ 84.0(65.3-93.6) & 86.2(69.4-94.5) \\ 94.0(85.8-97.7) & 93.4(84.3-97.4) \\ 46.6(32.9-60.9) & 71.4(54.9-83.7) \\ 4.6(1.8-11.3) & 5.32(2.15-13.13) \\ 0.3(0.23-0.5) & 0.18(0.10-0.32)\end{array}$

Data are presented with $95 \%$ confidence intervals. PPV: positive predictive value; NPV: negative predictive value. LR+: positive likelihood ratio; LR-: negative likelihood ratio.

and likely the determinant factor, is that all types of hypersensitivity pneumonitis were included, regardless of the causal agent. As would be expected, our group has considerable experience in performing SIC with avian proteins [10] and fungi [11, 27, 28], but much more limited practice with the less common causal agents of hypersensitivity pneumonitis. This was illustrated when only patients with hypersensitivity pneumonitis caused by exposure to birds or fungi were analysed: there were only 10 false-negative results and the sensitivity of the test rose to $85 \%$. Finally, there are a high percentage of patients with usual interstitial pneumonia in the false-negative group (31.8\%). These chronic fibrotic patients tend to have lower inflammatory cell infiltrates, indicating perhaps that the test may not be useful for this subgroup [29]. We do not know the effect that age and smoking habit can have on the positivity or negativity of the SIC.

One of the main problems for definite implementation of SIC in the diagnosis of hypersensitivity pneumonitis stems from the lack of a well-defined method for carrying out the test. This may also have contributed to the differences in false-negative rates. Three methods have been proposed for SIC. First, some authors hold that natural workplace or home exposure is a reasonable way to provoke symptoms or manifest deterioration of functional parameters in uncertain cases of hypersensitivity pneumonitis [4]. Secondly, it may be useful to try to reproduce the working or environmental conditions in a challenge chamber. This method has not been widely used [14,30], and although it does not conclusively identify the causal agent, it may be easier to standardise and possibly safer. The third method consists of using an aerosolised extract material of the suspected causal agent. This may be the most commonly used method, with one of the first descriptions published by Williams [31] in 1963. It is the method we apply when the suspected agents are avian proteins or fungi [7-9]. It is important to mention that the SIC method used by our group is focussed on achieving the correct diagnosis while submitting the patient to the least possible
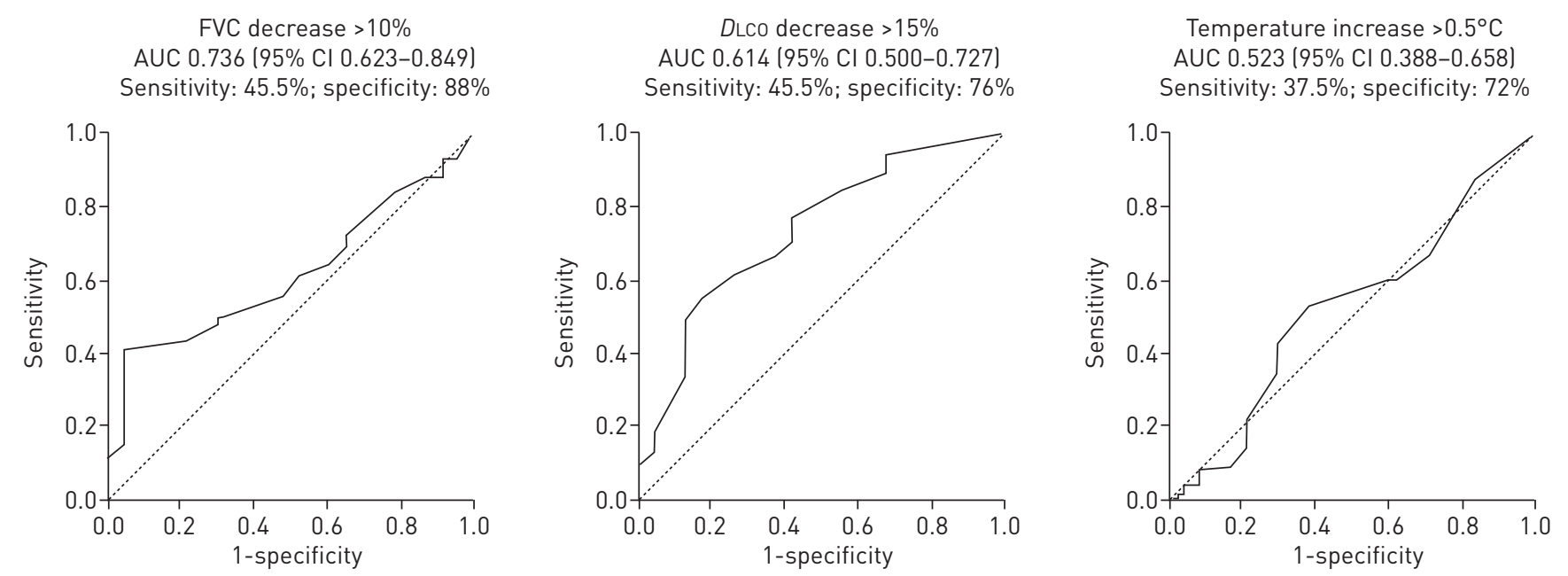

FIGURE 2 Receiver operating characteristic curves constructed to determine the most relevant differences in forced vital capacity (FVC), diffusing capacity of the lung for carbon monoxide (DLCO), and temperature before and after specific inhalation challenge for predicting hypersensitivity pneumonitis. AUC: area under the curve. 
risk. Thus, the level of antigen exposure is probably lower than that used by other groups. For example, maximum exposure in the studies of OTANI et al. [9] was $680 \mu \mathrm{g}$ of protein, whereas our group uses a maximum dose of $200 \mu \mathrm{g}$ protein. The rationale is that fewer respiratory and systemic symptoms will be produced, and that possible pulmonary function abnormalities will be rapidly reversible.

Related with this last observation, another critical aspect is how to establish a positive outcome in SIC. Whereas HENDRICK et al. [7] and OTANi et al. [9] basically considered the test positive when symptoms and signs consistent with an influenza-like illness developed, other groups place more importance on objective measures than on clinical aspects. RAMírEZ-VENEGAS et al. [8] considered the test positive when a decrease $>16 \%$ in $\mathrm{FVC},>3 \mathrm{mmHg}$ in $\mathrm{PaO}_{2},>3 \%$ in arterial oxygen saturation, or a body temperature increase $>0.5^{\circ} \mathrm{C}$ occurred. Our group, however, has assigned greater value to pulmonary function studies $[10,13]$. When the criterion of $>0.5^{\circ} \mathrm{C}$ body temperature increase is combined with an FVC decrease $>15 \%$, LCO decrease $>20 \%$, or FVC between $10 \%$ and $15 \%$, the test shows good sensitivity, particularly in the study of avian or fungal antigens. When these parameters were tested separately, specificities were good, but sensitivities were low. For this reason, we believe that combined assessment of these parameters provides the best diagnostic yield.

Another important aspect when recommending SIC in the diagnosis of hypersensitivity pneumonitis is the small number of associated adverse effects. Only nine patients experienced such effects, which were transient in all cases, and only three patients required oral corticosteroid administration. These secondary effects occurred in younger patients with higher baseline DLCO levels, an observation that should alert to the need for special precautions (e.g. decrease the dose of inhaled antigen and hospitalise the patient) in this patient group. The fact that complications presented in individuals with a nearly normal DLCO may be because these patients are more prone to note clinical changes than those in whom DLCO is already decreased and baseline symptoms may be present. It is important to mention that in our protocol, SIC is only contraindicated when the FVC and DLCO are less than 50\% and 40\%, respectively, of the theoretical value [13]. Some authors believe that SIC should not be performed under any circumstances because the test itself may sensitise the patient and cause illness [32]. This has not been observed in any patients undergoing SIC in whom a diagnosis other than hypersensitivity pneumonitis was ultimately established. Nor has it been observed in other studies in which healthy control subjects underwent SIC $[7,8,10]$.

This study has the limitations of a retrospective design. Although all patients were studied according to the same protocol, SIC was performed for diagnostic purposes and not in the context of a study designed to determine its performance. This limitation may have been compensated by the fact that the definite diagnosis of the disease was established at the time of the study by two independent observers who took into consideration the patients' follow-up data in addition to the proposed diagnostic criteria for hypersensitivity pneumonitis [2, 33]. This fact practically assures the diagnosis and likely enables establishment of the usefulness of the test in actual clinical practice. Another possible limitation is the fact that the data for sensitivity and specificity were calculated in the context of diagnosis of hypersensitivity pneumonitis, and not in the context of the differential diagnosis of interstitial lung diseases. Future studies should be designed to determine the sensitivity and specificity of SIC for the differential diagnosis of interstitial lung diseases.

In conclusion, the results of the present study show that SIC could be useful in the diagnosis process of hypersensitivity pneumonitis. In fact, a positive result is practically diagnostic for this disease, with good sensitivity. It has also proven to be a safe test, with few adverse effects. Nonetheless, efforts should be made to standardise the way in which SIC is performed, particularly when the suspected causal agent is not avian or fungal proteins, and the interpretation of the results.

\section{References}

Selman M. Hypersensitivity pneumonitis: a multifaceted deceiving disorder. Clin Chest Med 2004; 25: 531-547.

Schuyler M, Cormier Y. The diagnosis of hypersensitivity pneumonitis. Chest 1997; 111: 534-536.

Selman M. Hypersensitivity pneumonitis. In: Schwarz MI, King TE, eds. Interstitial Lung Disease. Shelton, People's Medical Publishing House, 2011; pp. 597-635.

Costabel U, Bonella F, Guzman J. Chronic hypersensitivity pneumonitis. Clin Chest Med 2012; 33: 151-163.

Lacasse Y, Girard M, Cormier Y. Recent advances in hypersensitivity pneumonitis. Chest 2012; 142: 208-217.

Selman M, Pardo A, King TE Jr. Hypersensitivity pneumonitis. Insights in diagnosis and pathobiology. Am J Respir Crit Care Med 2012; 186: 314-324.

Hendrick DJ, Marshall R, Faux JA, et al. Positive "alveolar" response to antigen inhalation provocation test: their validity and recognition. Thorax 1980; 35: 415-427.

8 Ramírez-Venegas A, Sansores RH, Perez-Padilla R, et al. Utility of a provocation test for diagnosis of chronic pigeon breeder's disease. Am J Respir Crit Care Med 1998; 158: 862-869.

9 Ohtani Y, Kojima K, Sumi Y, et al. Inhalation provocation test in chronic bird fancier's lung. Chest 2000; 118 : 1382-1389.

10 Morell F, Roger A, Reyes L, et al. Bird fancier's lung. A series of 86 patients. Medicine (Baltimore) 2008; 87: 110-130. 
11 Cruz MJ, Morell F, Roger A, et al. Hypersensitivity pneumonitis in construction plasterers (espartosis): study of 20 patients. Med Clin (Barc) 2003; 120: 578-583.

12 Uranga A, Sánchez-Ortiz M, Morell F, et al. Hypersensitivity pneumonitis due to isocyanates: lung function, clinical and radiological characteristics. Arch Bronconeumol 2013; 49: 169-172.

13 Muñoz X, Morell F, Cruz MJ. The use of specific inhalation challenge in hypersensitivity pneumonitis. Curr Opin Allergy Clin Immunol 2013; 13: 151-158.

14 Sogo A, Morell F, Muñoz X. Hypersensitivity pneumonitis associated with the use of a steam iron. Arch Bronconeumol 2009; 45: 258-259.

15 Toribio R, Cruz MJ, Morell F, et al. Hypersensitivity pneumonitis related to medium-density fiberboard. Arch Bronconeumol 2012; 48: 29-31.

16 Quanjer PH, Tammeling GJ, Cotes JE, et al. Lung volumes and forced ventilatory flows. Report Working Party Standardization of Lung Function Tests, European Community for Steel and Coal, Official Statement of the European Respiratory Society. Eur Repir J 1993; 6: 5-40.

17 Cotes JE, Chinn DJ, Quanjer PH, et al. Standardization of the measurement of transfer factor (diffusing capacity). Report Working Party Standardization of Lung Function Tests, European Community for Steel and Coal, Official Statement of the European Respiratory Society. Eur Respir J 1993; 6: 41-52.

18 Roca J, Sanchis J, Augusti-Vidal A, et al. Spirometric reference values from a Mediterranean population. Bull Eur Physiopathol Respir 1986; 22: 217-224.

19 Miller MR, Hankinson J, Brusasco V, et al. ATS/ERS Task Force. Standardisation of spirometry. Eur Respir J 2005; 26: 319-338.

20 MacIntyre N, Crapo RO, Viegi G, et al. Standardisation of the single-breath determination of carbon monoxide uptake in the lung. Eur Respir J 2005; 26: 720-735.

21 Altman DG, Bland JM. Diagnostic tests. 1: Sensitivity and specificity. BMJ 1994; 308: 1552.

Altman DG, Bland JM. Diagnostic tests 2: Predictive values. BMJ 1994; 309: 102.

Deeks JJ, Altman DG. Diagnostic tests 4: Likelihood ratios. BMJ 2004; 329: 168-169.

Wilson EB. Probable inference, the law of succession, and statistical inference. J Am Stat Assoc 1927; 22: 209-212. Altman DG, Bland JM. Diagnostic tests 3: receiver operating characteristic plots. BMJ 1994; 309: 188.

Sanchez-Ortiz M, Cruz MJ, Viladrich M, et al. Cryptogenic organizing pneumonia due to ortho-phenylenediamine. Respir Med CME 2011; 4: 164-165.

27 Morell F, Roger A, Cruz MJ, et al. Suberosis. Clinical study and new etiologic agents in a series of eight patients. Chest 2003; 124: 1145-1152.

28 Morell F, Cruz MJ, Gomez FP, et al. Chacinero's lung - hypersensitivity pneumonitis due to dry sausage dust. Scand J Work Environ Health 2011; 37: 349-356.

29 Ohtani Y, Saiki S, Kitaichi M, et al. Chronic bird fancier's lung: histopathological and clinical correlation. An application of the 2002 ATS/ERS consensus classification of the idiopathic interstitial pneumonias. Thorax 2005; 60: 665-671.

30 Baur X. Hypersensitivity pneumonitis (extrinsic allergic alveolitis) induced by isocyanates. J Allergy Clin Immunol 1995; 95: 1004-1010.

31 Williams JV. Inhalation and skin tests with extracts of hay and fungi in patients with farmer's lung. Thorax 1963; 18: $182-196$.

32 Richerson HB, Bernstein IL, Fink JN, et al. Guidelines for the clinical evaluation of hypersensitivity pneumonitis. Report of the Subcommittee on Hypersensitivity Pneumonitis. J Allergy Clin Immunol 1989; 84: 839-844.

33 Lacasse Y, Selman M, Costabel U, et al. Clinical diagnosis of hypersensitivity pneumonitis. Am J Respir Crit Care Med 2003; 168: 952-958. 